\title{
Accelerometer-Based Automated Counting of Ten Exercises without Exercise-Specific Training or Tuning
}

\author{
Samuel Zelman $\mathbb{D}^{1},{ }^{1}$ Michael Dow, ${ }^{1}$ Thasina Tabashum $\mathbb{D}^{2},{ }^{2}$ Ting Xiao $\mathbb{D},{ }^{2}$ \\ and Mark V. Albert $\mathbb{D}^{2,3,4}$ \\ ${ }^{1}$ Illinois Math and Science Academy, Aurora, IL 60506, USA \\ ${ }^{2}$ Department of Computer Science and Engineering, University of North Texas, Denton, TX 76203, USA \\ ${ }^{3}$ Department of Biomedical Engineering, University of North Texas, Denton, TX 76203, USA \\ ${ }^{4}$ Department of Physical Medicine and Rehabilitation, Northwestern University Feinberg School of Medicine, Chicago, IL 60611, \\ USA
}

Correspondence should be addressed to Mark V. Albert; mark.albert@unt.edu

Received 17 May 2020; Revised 18 August 2020; Accepted 29 September 2020; Published 10 October 2020

Academic Editor: Fabrizio Taffoni

Copyright (C) 2020 Samuel Zelman et al. This is an open access article distributed under the Creative Commons Attribution License, which permits unrestricted use, distribution, and reproduction in any medium, provided the original work is properly cited.

\begin{abstract}
Measuring physical activity using wearable sensors is essential for quantifying adherence to exercise regiments in clinical research and motivating individuals to continue exercising. An important aspect of wearable activity tracking is counting particular movements. One limitation of many previous models is the need to design the counting for a specific exercise. However, during physical therapy, some movements are unique to the patient and also valuable to track. To address this, we create an automatic repetition counting system that is flexible enough to measure multiple distinct and repeating movements during physical therapy without being trained on the specific motion. Accelerometers, using smartphones, were attached to the body or held by participants to track repetitive motions during different exercises. 18 participants completed a series of 10 exercises for 30 seconds, including arm circles, bicep curls, bridges, sit-ups, elbow extensions, leg lifts, lunges, push-ups, squats, and upper trunk rotations. To count the repetitions of each exercise, we apply three analysis techniques: (a) threshold crossing, (b) threshold crossing with a low-pass filter, and (c) Fourier transform. The results demonstrate that arm circles and push-ups can be tracked well, while less periodic and irregular motions such as upper trunk rotations are more difficult. Overall, threshold crossing with low-pass filtering achieves the best performance among these methods. We conclude that the proposed automatic counting system is capable of tracking exercise repetition without prior training and development for that activity.
\end{abstract}

\section{Introduction}

Physical therapy is a key strategy to improve mobility and quality of a patient's life after injuries, surgeries, and other debilitating events [1]. Physical therapy sessions take place in clinical settings with licensed physical therapists that help to instruct and encourage patients to increase comfort and mobility [2]. Unfortunately, these sessions may occur infrequently and therefore provide insufficient information about each patient's activities and efforts. Evaluation of physical therapy has always been considered as a challenge in research [3, 4]. An automated system for tracking exercises at home can play a vital role in improving patient's motivation and adherence to prescribed repetitive exercises as well as help to track and encourage patient's movements with minimum guidance from a physical therapist.

Wearable devices are the body-worn technologies that can perform computing functionalities during execution of physical tasks [5]. Such knowledge of users' physical status provides huge scope for utilizing wearable devices to assist clinicians to evaluate physical activity and can be applied to track posture in the clinical population [6]. Accelerometers are common sensors which are integrated in wearable technologies, and the use of accelerometers has escalated significantly over the past decade in the health-monitoring research area [7]. Using signal features extracted by improved modern accelerometers allowed experts to utilize those information in clinical science [8]. Despite the fact that 
the use of accelerometers has increased exponentially, reliable assessment of physical activity is still needed to explore [9]. However, it is also important to consider the technologies that are easily accessible and part of our daily life, such as cellphones. Most smartphones now incorporate a threeaxis accelerometer sensor, and phone accelerometers are now used to perform activity recognition [10]. Implementing a simple step count can be achieved easily with the latest smartphones, but the activity tracking system without depending on labeled training data offers substantial research scope. There have already been some studies of activities on quantifying and recognizing using mobile phones [11-13], but this technology has not been fully examined for unknown activity.

In the context of physical therapy, the exercises depend on the specific conditions of every patient. Physical therapy exercises can differ significantly from one exercise to the next, which makes developing tailored recognition strategies challenging when the type of activities are unknown. One limitation with activity recognition models is that most of them are pretrained. People are instructed to make a particular movement (steps, walking, running, etc.), and data are acquired on that movement; then, offline analysis is performed to create a model to specifically measure that particular movement. Generally, machine learning techniques perform well on this kind of task when large volumes of data are available [14]. However, pretrained models require sufficient data for that particular movement to train and validate; this is difficult for unique instructed movements which can happen in physical therapy rehabilitation. Furthermore, periodic signals can offer a window of opportunities in various research problems. Many previous studies required large amounts of labeled data to achieve proper performance to count exercises. For example, to build RecoFit, a total 114 participants were recorded in over 146 sessions [15]. To address the concern of availability of quality data, we used the periodic signals and proposed methods that are adaptive. Our approach does not need labeled training data to perform automatic counting and it can be integrated with future work that aims to address realtime scenarios of repetition count.

In this study, we apply three straightforward analysis techniques to count the periodic motions. Ten different exercises were performed to assess not only the analysis strategies but also compare the quality of results for each exercise. Repeated exercises can help to perform automatic counting by using a repetitive structure of signals.

\section{Materials and Methods}

Smartphones with accelerometers have been placed on 18 subjects, aged 15-25 years, who completed a series of 10 different exercises for 30 seconds each, repeating each exercise twice, and we took the average of the two readings; however, differences in the count were rare. 7 females and 11 males participated in the study. These activities were arm circles, bicep curls, bridges, sit-ups, elbow extensions, leg lifts, lunges, push-ups, squats, and upper trunk rotations. We chose 10 common exercises that can be performed safely allowing untrained participants to execute the exercises properly. The exercises were also selected to involve a combination and coordination of muscles across the body. Three standard analysis techniques have been compared to count the number of repetitions of each activity: threshold crossing, threshold crossing with a low-pass filter, and a Fourier transform approach.

To collect data for this experiment, LG Optimus S smartphone accelerometers were used. The Sensor Log application (Sensor Log) recorded acceleration on 3 axes. The smartphone was either held by participants or strapped onto them, based on the exercise they were performing. The location of the smartphone for each exercise is listed in Table 1. Researchers found a similar level of accuracy for detecting everyday activities from a sensor placed in five different locations [16]. We decided to place the device according to what type of exercise, e.g., arm circle and elbow extension on hand and sit ups on the chest. The number of repetitions of each exercise was recorded by both the participant and the observer. Each exercise was repeated, and its reading was removed from analysis if the number of counted repetitions by participants and observers were not the same. Between each exercise, the participants were allowed an optional one-minute break. The smartphone's sensors were started at the beginning of each activity and were turned off at the end of the activity, to eliminate nonexercise data. The collected accelerometer data from smartphones were converted into magnitudes of acceleration and then trimmed manually only at the beginning and end of the 30-second session to obtain only the exercises and filter out nonactivity data. A sign multiplication was applied to the magnitude of the accelerometer in order to avoid a half-wave rectification of the signal. If the signal was in the direction of gravity (using a long-timescale moving average), the signal was positive and if opposite, it was negative. The experiments have been approved by the Illinois Math and Science Academy Institutional Review Board. It has been confirmed that all experiments were performed under relevant guidelines and regulations.

Three methods of estimating activity counts including threshold crossing, threshold crossing with a low-pass filter, and a Fourier transform approach have been used. Each method was written in Python, using the NumPy and Sci$\mathrm{Py}+$ libraries. Each analysis technique is as follows.

2.1. Threshold Crossing. This technique has been utilized to calculate activity counts by tuning the threshold line position at two-third of the range between the minimum and maximum. When the accelerometer data cross the line in a positive direction, one count would be added. After adding each count, a refractory period of 0.1 seconds has occurred to prevent counting another repetition needlessly.

2.2. Threshold Crossing with Low-Pass Filtering. The technique was identical to the threshold crossing technique, except for the application of a Butterworth filter to minimize the impact of high-frequency noise. The low-pass filter 
TABLE 1: Location where the smartphone is placed and how it is held by the individual during the exercise.

\begin{tabular}{lcc}
\hline Exercise & Location & Holding methods \\
\hline Arm circle & Hand & Held in hand: random orientation \\
Bicep curl & Hand & Held in hand: random orientation \\
Bridge & Waist & Pouch \\
Crunch & Chest & Held in hands with hands on positioned on chest \\
Elbow extension & Hand & Held in hand: random orientation \\
Lower trunk rotation & Waist & Pouch \\
Lunge & Waist & Pouch \\
Push ups & Waist & Pouch \\
Squats & Waist & Pouch \\
Upper trunk rotation & Waist & Pouch \\
\hline
\end{tabular}

method was performed using a moving average filter with the formula $A_{i+1}=0.9 A_{i}+0.1 X_{i}$.

2.3. Fourier Transform. This method used a standard Fourier frequency decomposition, where the number of repetitions was calculated using the dominant frequency based on the length of time of the data collection. Count $=$ frequency $*$ time.

The threshold level was the third quartile $(30 \%$ percentile) of the magnitude distribution, which was chosen by maximizing accuracy using a grid sampling on a convenience sample for methods 2 and 3.

\section{Results}

The general data analysis approach is depicted in Figure 1. Given this approach, it is expected that exercises with more periodic and large motions such as squats, push-ups, and arm circles will likely be counted with higher accuracy. The less periodic and smaller range of motion movements such as bridges, upper trunk rotations, and bicep circles are less accurately measured. Table 2 records the accuracy of each method with the type of exercise performed by individuals. From the table, it can be seen that arm circles are counted well even by using the simple threshold method, likely due to a very small noise in the data and the periodic nature of the motion. On the other hand, upper trunk rotations were more difficult to count, especially with the simple threshold crossing method.

Accelerometer data were recorded for 30 seconds along with manual counts of the exercises. Figure 2 demonstrates the 3-axis accelerometer data and the acceleration magnitude for arm circles, push-ups, and upper trunk rotations noting the expected high-, medium-, and low-accuracy movements. We can observe in these magnitudes (Table 2) that exercises with more identifiable repetition in the signal and large motions such as squats, push-ups, and arm circles will likely be counted with higher accuracy. The less repetitive and smaller range of motion movements such as bridges, upper trunk rotations, and bicep circles may be less accurately measured.

In general, the results demonstrate that threshold crossing with a low-pass filter is the most effective counting method, with an average root mean square error of 8.69, noting a high variation depending on the movement being tracked. Fourier transform also performs reasonably well, with a root mean square error of 9.00. Threshold crossing without filtering does not perform well based on both of the roots mean square error of 13.38 as expected. Squats are the easiest exercise to count, whereas upper trunk rotations are the most difficult. Thus, we can conclude that periodic motions with substantial ranges are easiest to count.

\section{Discussion}

The goal of this study was to determine the accuracy of simple methods of counting repetitions from 10 different activities using an accelerometer. We applied three different methods of counting which include threshold crossing, threshold crossing with low-pass filtering, and the Fourier transform. We find that the best results for all counts belong to the large, periodic motion exercises, as expected.

To count motions accurately in practice, we need to address several different challenges. Since accelerometer data varied depending on the exercise being studied, we did not tailor the counting method to each specific activity. The chosen counting methods are flexible and can be used on any patient, even those with abnormal movement patterns. This flexible approach has an additional benefit of making these methods more robust; the accuracy achieved without utilizing automatic segmentation or recognition. It can count activities without having prior knowledge of the type of activity. Notably, there are limitations to this overall strategy. We are unable to count more than one distinct activity taking place in the same period, which can be particularly problematic when accounting for prolonged moments of rest between motions. Another constraint is that the experiment was conducted in a limited age group (between 15 and 25). For older people, it might be different and more challenging. In this case, the duration of the reading for each exercise should be considered as it will take more time to complete each repetition and there might be more noise in accelerometer data. Further critical decision is the location of an accelerometer.

Machine learning-based activity recognition models can provide accurate counts when the particular activity being counted is pretrained. There are numerous studies to show the reliability and accuracy of accelerometer-based activity measurements [17]. Fall detection relies on accurate training data $[18,19]$. ReadySteady, an accelerometer-based mobile 

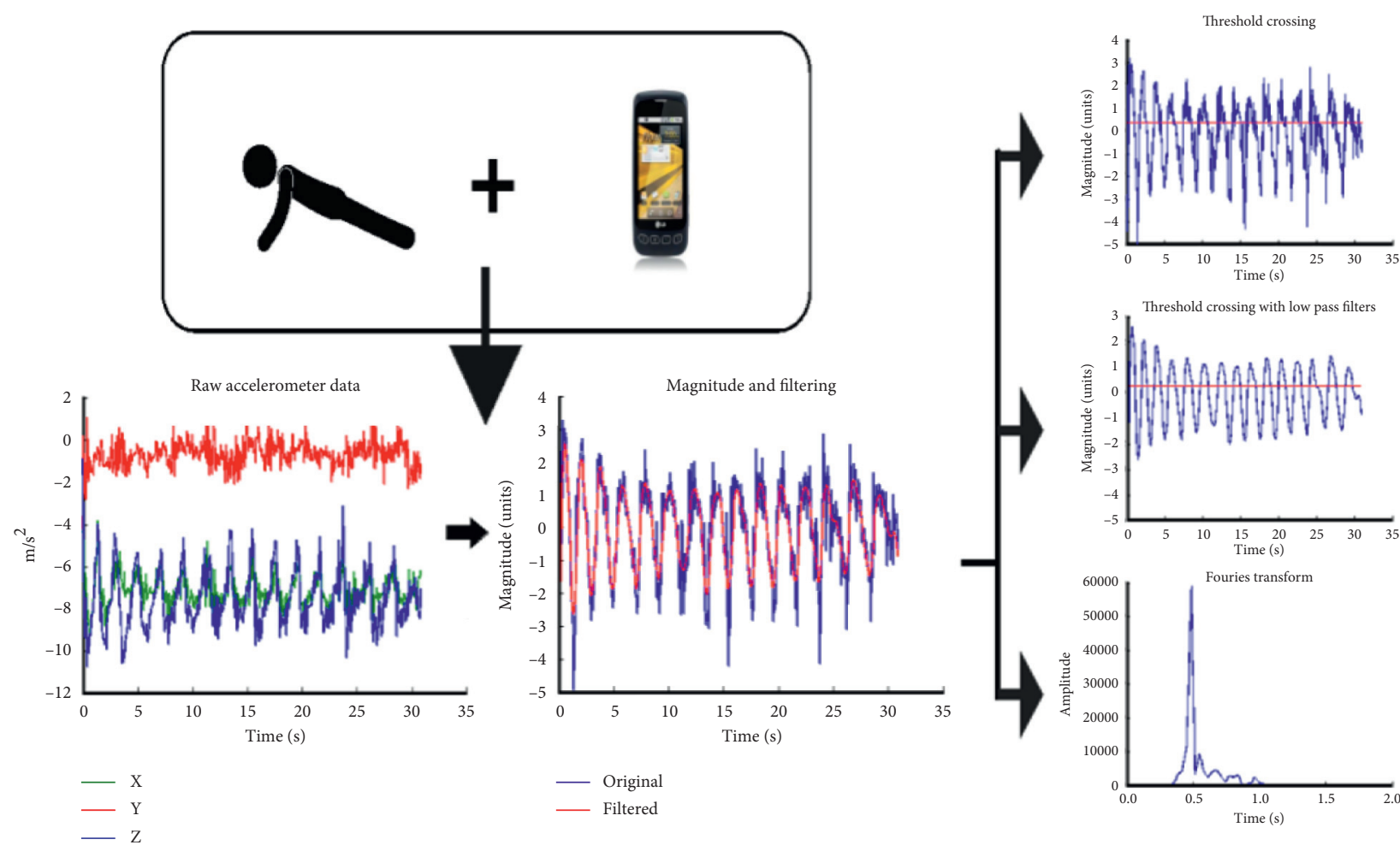

FIGURE 1: The data from the smartphone accelerometer is analyzed by applying three different counting methods. The 3-axis accelerometer reading is converted to a magnitude and processed further by (top) counting when the function crosses a threshold (middle) (threshold crossing) but after smoothing the magnitude (bottom) using the frequency indicated by a peak in the Fourier analysis to estimate count over time.

TABLE 2: Root mean square error (RMSE) of counts for each analysis technique.

\begin{tabular}{|c|c|c|c|c|}
\hline Exercise & Threshold crossing & Threshold with low pass & Fourier & Avg RMSE \\
\hline Arm circle & 1.99 & 9.65 & 8.08 & 7.36 \\
\hline Bicep curl & 16.70 & 7.36 & 12.29 & 12.71 \\
\hline Bridge & 13.94 & 14.39 & 7.07 & 12.27 \\
\hline Crunch & 7.32 & 8.56 & 12.18 & 9.58 \\
\hline Elbow extension & 13.70 & 10.20 & 10.57 & 11.59 \\
\hline Lower trunk rotation & 13.47 & 9.97 & 7.45 & 10.59 \\
\hline Lunge & 16.95 & 1.83 & 0.76 & 9.85 \\
\hline Push-ups & 10.53 & 1.39 & 3.70 & 6.49 \\
\hline Squats & 10.71 & 1.50 & 0.93 & 6.27 \\
\hline Upper trunk rotation & 19.06 & 9.77 & 14.11 & 14.81 \\
\hline Avg RMSE & 13.38 & 8.69 & 9.00 & \\
\hline
\end{tabular}

Threshold crossing with a low-pass filter performs best with an average root mean square error of 8.69 , with the Fourier transform being close with 9.00 .

application for activity assessment, has been developed, which shows that the system can differentiate the intensity of any activity, for example, sitting, driving, and walking [20]. A previous study analyzed the repetition accuracy of weight training and calisthenics through an arm-worn accelerometer [15]. Additionally, activity recognition can be improved through the use of dynamic state estimators such as hidden Markov models [21, 22]. The use of segmentation and activity recognition allowed the researchers to improve repetition count accuracy, as well as identifying exercise data from nonexercise data. They also discussed how individual learning models can improve counting accuracy for the activities, once activities are segmented and classified.
Further studies demonstrated using a routine tracking of health can motivate and encourage positive health behaviors $[23,24]$. For instance, researchers at Stanford found that pedometer users had a $26.9 \%$ increase in activity over baseline [25]. Cadmus-Bertham illustrates that supplying Fitbits to postmenopausal women led to increased physical activity, over a period of 16 weeks [26]. Having a simple way to collect detailed exercise data at home can help physical therapists tailor their sessions in the clinic, which provides a large benefit to physical care. In summary, we evaluated the effectiveness of three different analysis methods in the counting of exercises. The error rate is higher than it is expected to be considered usable, primarily as there was no 

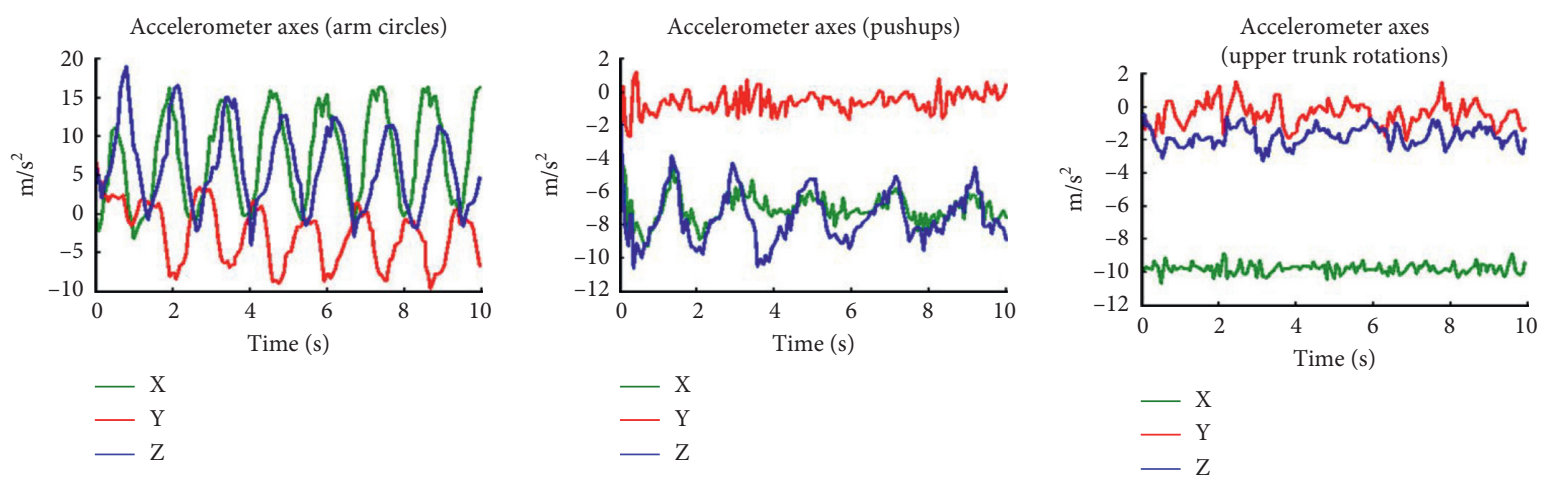

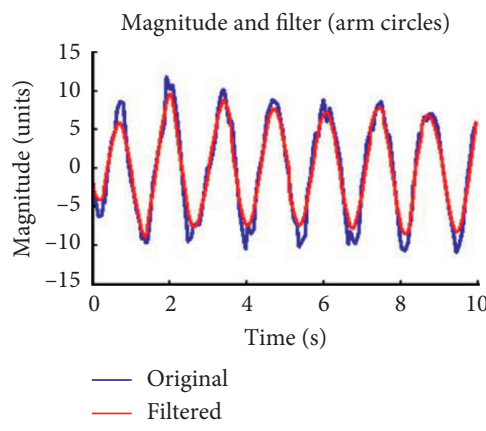

(a)

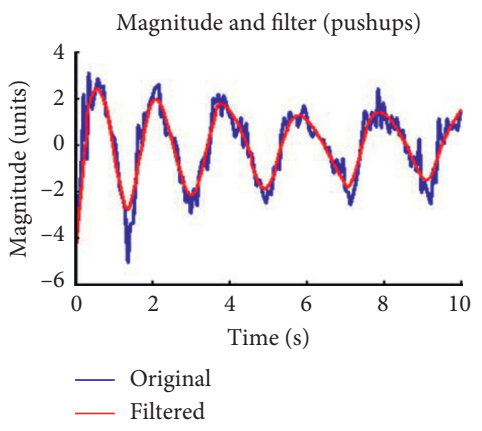

(b)

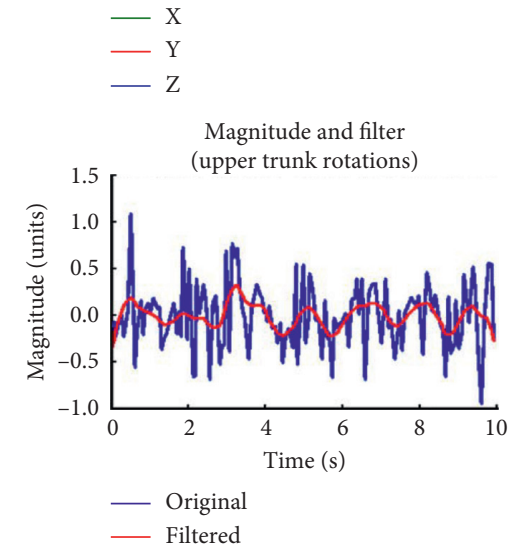

(c)

FIGURE 2: An observation of acceleration data for three different types of motions such as (a)arm circles (b) upper trunk rotation (c) bridges, representing more to less periodic motion, respectively.

tuning to individual activities. The location of the accelerometer is one concern. It is important to note that without any prior knowledge of the data, we can see a pattern of how repetitive exercises can be tracked using our method. The most effective method for each task varied, due to different amounts of noise and different levels of periodicity. Threshold crossing works well with arm circles and crunches. After adding a smoothing filter to remove noise and high-frequency motion threshold crossing also works well for lunges, pushups, and squats, with higher general performance in all activities other than arm circles and crunches. The Fourier transform approach works exceptionally well for lunges and squats but generally performs poorly overall. These methods can serve as effective baselines for unspecified periodic activity counting.

In the future, we plan to perform and test our method on clinical populations including older subjects and individuals with disabilities. In addition, machine learning methods can be explored; this is an extensive approach used in human activity recognition, but commonly, it is applied to create pretrained models for specific movements. Variations of training paradigms will be explored to use machine learning without identifying the type of movement.

\section{Conclusion}

In this study, we performed three analysis techniques to achieve automatic counting of unseen exercises. The repetitive motions of ten exercises of eighteen participants were evaluated through (a) threshold crossing, (b) threshold crossing with a low-pass filter, and (c) Fourier transform. From the result, we can conclude that less periodic activities are more prone to error, whereas the periodic motions can be tracked easily using our approach. This study can be applied to perform and evaluate exercises at home with minimal help from a physical therapist. Therefore, automatic counting methods help patients to save time and money by decreasing the number of rehabilitation sessions and encouraging therapy compliance. This study would have an extended effect on physical therapy and, ultimately, such tracking can provide clinical benefit to patients and therapists.

\section{Data Availability}

The data used to support the findings of this study have not been made available.

\section{Ethical Approval}

The Illinois Math and Science Academy Institutional Review Board approved this study.

\section{Consent}

Informed, written consent was acquired for all subjects.

\section{Conflicts of Interest}

The authors declare that they have no conflicts of interest. 


\section{Acknowledgments}

The authors would like to thank the IMSA SIR (Student Research and Inquiry) program for supporting the data collection of this effort and the students and faculty of the Loyola University Chicago Computer Science Summer Research program for their help in designing and validating this research effort. The authors would also like to thank Pinky Sindhu and Laurynas Kalesinskas for their assistance in assembling the resources for this paper.

\section{References}

[1] E. Laakso, A. Cantlay, and A. J. McAuliffe, "The impact of physiotherapy intervention on functional independence and quality of life in palliative patients," Cancer Forum, vol. 27, p. $15,2003$.

[2] L. A. Harvey, "Physiotherapy rehabilitation for people with spinal cord injuries," Journal of Physiotherapy, vol. 62, no. 1, pp. 4-11, 2016.

[3] C. E. Tudor-Locke and A. M. Myers, "Challenges and opportunities for measuring physical activity in sedentary adults," Sports Medicine, vol. 31, no. 2, pp. 91-100, 2001.

[4] S. H. J. Keus, M. Munneke, M. J. Nijkrake, G. Kwakkel, and B. R. Bloem, "Physical therapy in Parkinson's disease: evolution and future challenges," Movement Disorders, vol. 24, no. 1, pp. 1-14, 2009.

[5] C. Randell, Wearable Computing: A Review, Research Gate Department of Computer Science, University of Bristol, Bristol, UK, 2005, http://www.academia.edu/download/ 1738742/3zb5d09sg01m7xms.pdf.

[6] M. V. Albert, I. Shparii, and X. Zhao, "The applicability of inertial motion sensors for locomotion and posture," in Locomotion and Posture in Older Adults: The Role of Aging and Movement Disorders, F. A. Barbieri and R. Vitório, Eds., pp. 417-426, Springer International Publishing, Berlin, Germany, 2017.

[7] P. S. Freedson and K. Miller, "Objective monitoring of physical activity using motion sensors and heart rate," Research Quarterly for Exercise and Sport, vol. 71, no. 2, pp. S21-S29, 2000.

[8] R. P. Troiano, J. J. McClain, R. J. Brychta, and K. Y. Chen, "Evolution of accelerometer methods for physical activity research," British Journal of Sports Medicine, vol. 48, no. 13, pp. 1019-1023, 2014.

[9] R. P. Troiano, "A timely meeting: objective measurement of physical activity," Medicine \& Science in Sports \& Exercise, vol. 37, no. 11, pp. S487-S489, 2005.

[10] J. R. Kwapisz, G. M. Weiss, and S. A. Moore, "Activity recognition using cell phone accelerometers," ACM SigKDD Explorations Newsletter, vol. 12, no. 2, pp. 74-82, 2011.

[11] W. W. Myo, W. Wettayaprasit, and P. Aiyarak, "A more reliable step counter using built-in accelerometer in smartphone," Indonesian Journal of Electrical Engineering and Computer Science, vol. 12, no. 2, p. 775, 2018.

[12] M. Mladenov and M. Mock, "A step counter service for Javaenabled devices using a built-in accelerometer," in Proceedings of the 1st International Workshop on Context-A ware Middleware and Services: Affiliated with the 4th International Conference on Communication System Software and Middleware (COMSWARE 2009), pp. 1-5, Dublin, Ireland, December 2009.
[13] M. V. Albert, S. Toledo, M. Shapiro, and K. Kording, "Using mobile phones for activity recognition in Parkinson's patients," Frontiers in Neurology, vol. 3, 2012.

[14] K. Chen, D. Zhang, L. Yao, B. Guo, Z. Yu, and Y. Liu, "Deep learning for sensor-based human activity recognition: overview, challenges and opportunities," 2020.

[15] D. Morris, T. S. Saponas, A. Guillory, and I. Kelner, "Recofit: using a wearable sensor to find, recognize, and count repetitive exercises," in Proceedings of the SIGCHI Conference on Human Factors in Computing Systems, pp. 3225-3234, Toronto, Canada, March 2014.

[16] I. Cleland, B. Kikhia, C. Nugent et al., "Optimal placement of accelerometers for the detection of everyday activities," Sensors, vol. 13, no. 7, pp. 9183-9200, 2013.

[17] C. U. Manohar, S. K. McCrady, Y. Fujiki, and I. T. Pavlidis, "Evaluation of the accuracy of a triaxial accelerometer embedded into a cell phone platform for measuring physical activity," Journal of Obesity \& Weight Loss Therapy, vol. 1, no. $1,2011$.

[18] M. V. Albert, K. Kording, M. Herrmann, and A. Jayaraman, "Fall classification by machine learning using mobile phones," PLoS One, vol. 7, no. 5, https://www.ncbi.nlm.nih.gov/pmc/ articles/pmc3346841/, 2012.

[19] N. Shawen, L. Lonini, C. K. Mummidisetty et al., "Fall detection in individuals with lower limb amputations using mobile phones: machine learning enhances robustness for real-world applications," JMIR mHealth and uHealth, vol. 5, no. 10, p. e151, 2017.

[20] M. Vankipuram, S. McMahon, and J. Fleury, "ReadySteady: app for accelerometer-based activity monitoring and wellness-motivation feedback system for older adults," AMIA Annual Symposium Proceedings, vol. 2012, pp. 931-939, 2012.

[21] M. V. Albert, A. Sugianto, K. Nickele et al., "Hidden Markov model-based activity recognition for toddlers," Physiological Measurement, vol. 41, no. 2, Article ID 025003, 2020.

[22] P. Sok, T. Xiao, Y. Azeze, and A. Jayaraman, "Activity recognition for incomplete spinal cord injury subjects using hidden markov models," IEEE Sensors Journal, vol. 18, no. 15, https://ieeexplore.ieee.org/abstract/document/8376012/, 2018.

[23] M. Figueiredo, C. Caldeira, Y. Chen, and K. Zheng, "Routine self-tracking of health: reasons, facilitating factors, and the potential impact on health management practices," Archive of AMIA Annual Symposium Proceedings, vol. 2017, pp. 706714, 2017.

[24] S. Consolvo, P. Klasnja, D. W. McDonald et al., "Flowers or a robot army? encouraging awareness \& activity with personal, mobile displays," in Proceedings of the 10th International Conference on Ubiquitous Computing, pp. 54-63, Seoul, Korea, September 2008.

[25] D. M. Bravata, C. Smith-Spangler, V. Sundaram et al., "Using pedometers to increase physical activity and improve health," The Journal of the American Medical Association, vol. 298, no. 19, pp. 2296-2304, 2007.

[26] L. A. Cadmus-Bertram, B. H. Marcus, R. E. Patterson, B. A. Parker, and B. L. Morey, "Randomized trial of a fitbitbased physical activity intervention for women," American Journal of Preventive Medicine, vol. 49, no. 3, pp. 414-418, 2015. 